\title{
Dual-Color Fluorescence Cross-Correlation Spectroscopy to Study Protein-Protein Interaction and Protein Dynamics in Live Cells
}

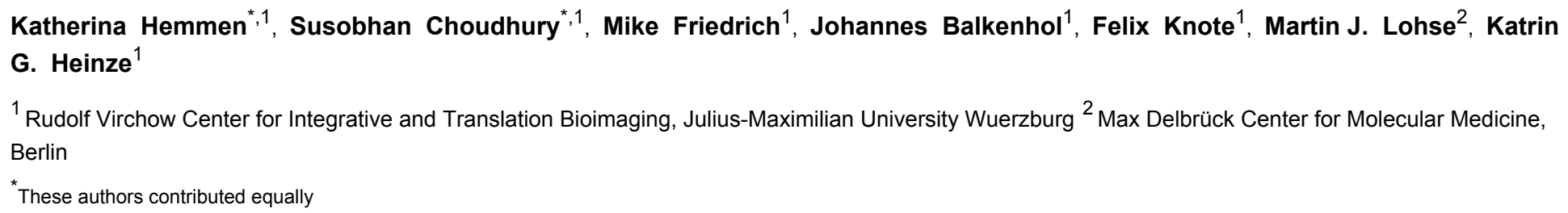

\section{Corresponding Author}

Katrin G. Heinze

katrin.heinze@uni-wuerzburg.de

\section{Citation}

Hemmen, K., Choudhury, S.,

Friedrich, M., Balkenhol, J., Knote, F., Lohse, M.J., Heinze, K.G. Dual-Color Fluorescence Cross-Correlation Spectroscopy to Study Protein-Protein Interaction and Protein Dynamics in Live Cells. J. Vis. Exp. (178), e62954, doi:10.3791/62954 (2021).

\section{Date Published}

December 11, 2021

DOI

$10.3791 / 62954$

URL

jove.com/video/62954

\section{Abstract}

We present a protocol and workflow to perform live cell dual-color fluorescence crosscorrelation spectroscopy (FCCS) combined with Förster Resonance Energy transfer (FRET) to study membrane receptor dynamics in live cells using modern fluorescence labeling techniques. In dual-color FCCS, where the fluctuations in fluorescence intensity represent the dynamic "fingerprint" of the respective fluorescent biomolecule, we can probe co-diffusion or binding of the receptors. FRET, with its high sensitivity to molecular distances, serves as a well-known "nanoruler" to monitor intramolecular changes. Taken together, conformational changes and key parameters such as local receptor concentrations and mobility constants become accessible in cellular settings.

Quantitative fluorescence approaches are challenging in cells due to high noise levels and the vulnerability of the sample. Here we show how to perform this experiment, including the calibration steps using dual-color labeled $\beta_{2}$-adrenergic receptor $\left(\beta_{2} A R\right)$ labeled with eGFP and SNAP-tag-TAMRA. A step-by-step data analysis procedure is provided using open-source software and templates that are easy to customize.

Our guideline enables researchers to unravel molecular interactions of biomolecules in live cells in situ with high reliability despite the limited signal-to-noise levels in live cell experiments. The operational window of FRET and particularly FCCS at low concentrations allows quantitative analysis at near-physiological conditions.

\section{Introduction}

Fluorescence spectroscopy is one of the main methods to quantify protein dynamics and protein-protein interactions with minimal perturbation in a cellular context. Confocal fluorescence correlation spectroscopy (FCS) is one of 
the powerful methods to analyze molecular dynamics as it is single-molecule sensitive, highly selective, and live-cell compatible ${ }^{1}$. Compared with other dynamicsoriented approaches, FCS has a broader measurable time range spanning from $\sim \mathrm{ns}$ to $\sim \mathrm{s}$, most importantly covering the fast time scales that are often inaccessible by imaging-based methods. Moreover, it also provides spatial selectivity so that membrane, cytoplasmic, and nucleus molecular dynamics can be easily distinguished ${ }^{2}$. Thus, molecular blinking, the average local concentration, and the diffusion coefficient can be quantitatively analyzed with FCS. Intermolecular dynamics such as binding become easily accessible when probing codiffusion of two molecular species in fluorescence crosscorrelation spectroscopy (FCCS) analysis ${ }^{3,4,5}$ in a dualcolor approach.

The main underlying principle in correlation spectroscopy is the statistical analysis of intensity fluctuations emitted by fluorescently labeled biomolecules diffusing in and out of a laser focus (Figure 1A). The resulting auto- or crosscorrelation functions then can be further analyzed by curve fitting to eventually derive the rate constants of interest. In other words, the statistical methods FCS and FCCS do not provide single molecule traces like in single particle tracking, but a dynamic pattern or "fingerprint" of a probed specimen with high temporal resolution. When combined with Förster resonance energy transfer (FRET), intramolecular dynamics such as conformational changes can be monitored at the same time in a common confocal setup ${ }^{5,6}$. FRET probes the distance of two fluorophores and is often referred to as a molecular "nanoruler". Energy transfer takes place only when the molecules are in close vicinity $(<10 \mathrm{~nm})$, the emission spectrum of the donor significantly overlaps with the absorption spectrum of the acceptor molecule, and the dipole orientation of the donor and acceptor is (sufficiently) parallel. Thus, the combination of FRET and FCCS provides a technique with very high spatio-temporal resolution. When spatial selectivity, sensitivity as well as live-cell compatibility is required, FRET-FCCS has obvious advantageous over other methods such as Isothermal Titration Calorimetry (ITC) ${ }^{7}$, Surface Plasmon Resonance (SPR) ${ }^{8}$, or Nuclear Magnetic Resonance (NMR) $)^{9,10}$ for measuring protein dynamics and interactions.

Despite the capabilities and promise of dual-color fluorescence cross-correlation spectroscopy (dc-FCCS), performing dc-FCCS in live cells is technically challenging due to the spectral bleed-through or crosstalk between the channels $^{3,4}$, the difference in the confocal volumes due to the spectrally distinct laser lines ${ }^{3,4,11}$, background signal, and noise or limited photostability of the samples ${ }^{12,13,14,15}$. The introduction of pulse interleaved excitation (PIE) to FCCS was an important tweak to temporally decouple the different laser excitations for reducing the spectral crosstalk between the channels ${ }^{16}$. Other correction methods to counter spectral bleed-through ${ }^{17,18,19}$ and background corrections have also been well-accepted ${ }^{17,18,19}$. For details and basics on FCS, PIE or FRET the reader is referred to the following references $^{2,4,6,16,20,21,22,23,24}$.

Here, all necessary calibration experiments and analysis along with the experimental results of a prototypical Gprotein coupled receptor, $\beta_{2}$-adrenergic receptor $\left(\beta_{2} A R\right)$, for three different scenarios are presented: (1) singlelabeled molecules carrying either a "green" (eGFP) or a "red" (SNAP-tag-based labeling) ${ }^{25}$ fluorophore; (2) a double-labeled construct, which carries an N-terminal SNAPtag and intracellular eGFP (NT-SNAP) [in this case, both labels are at the same protein. Thus $100 \%$ co-diffusion is expected]; and (3) a double-labeled sample, where both 
fluorophores are on the same side of the cell membrane (CTSNAP). It carries a C-terminal SNAP-tag and an intracellular eGFP. Here, again both labels are at the same protein with again $100 \%$ co-diffusion expected. As both labels are very close to each other, on the same side of the cell membrane, it shows the potential to observe FRET and anticorrelated behavior. All constructs were transfected in Chinese Hamster Ovary $(\mathrm{CHO})$ cells and later labeled with a red fluorescent substrate which is membrane-impermeable for the NT-SNAP construct and membrane-permeable for the CT-SNAP construct. Finally, simulated data exemplifies the influence of experimental parameters on the FRET-induced anticorrelation, and the effect of protein-protein interactions on the co-diffusion amplitude.

Thus, this protocol provides a complete guide to performing the combined FRET-FCCS in living cells to understand protein dynamics and protein-protein interactions while making aware of technical/physical artifacts, challenges, and possible solutions.

\section{Protocol}

\section{Experimental Protocol}

\section{Sample preparation}

NOTE: Perform cell seeding and transfection under sterile conditions.

1. Place a cleaned coverslip per well into a 6-well culture plate and wash three times with sterile phosphate-buffered saline (PBS).

NOTE: The coverslip cleaning protocol is detailed in

\section{Supplementary Note 1.}

2. To each well, add $2 \mathrm{~mL}$ of the complete cell culture medium containing phenol red (supplemented with
$10 \%$ fetal bovine serum (FBS), $100 \mu \mathrm{g} / \mathrm{mL}$ penicillin and $100 \mu \mathrm{g} / \mathrm{mL}$ streptomycin) and keep the plate aside.

3. Culture the $\mathrm{CHO}$ cells in the same medium containing phenol red at $37{ }^{\circ} \mathrm{C}$ and $5 \% \mathrm{CO}_{2}$. Wash the cells with $5 \mathrm{~mL}$ of PBS to remove the dead cells.

4. Add $2 \mathrm{~mL}$ of trypsin and incubate for $2 \mathrm{~min}$ at room temperature (RT).

5. Dilute the detached cells with $8 \mathrm{~mL}$ of medium containing phenol red and mix carefully by pipetting.

6. Count the cells in a Neubauer chamber and seed at a density of $1.5 \times 10^{5}$ cells/well in the 6 -well cell culture plate containing the coverslips (prepared in step 1.1.1-1.1.2).

7. Let the cells grow in an incubator $\left(37^{\circ} \mathrm{C}, 5 \% \mathrm{CO}_{2}\right)$ for $24 \mathrm{~h}$ in order to achieve approximately $80 \%$ confluency.

8. Dilute $2 \mu \mathrm{g}$ of the desired vector DNA (e.g., CTSNAP or NT-SNAP) and $6 \mu \mathrm{L}$ of the transfection reagent in two separate tubes, each containing 500 $\mu \mathrm{L}$ of the reduced-serum medium for each well and incubate for $5 \mathrm{~min}$ at RT.

9. Mix the two solutions together to obtain the transfection mixture and incubate it for $20 \mathrm{~min}$ at RT.

10. In the meantime, wash the seeded $\mathrm{CHO}$ cells once with sterile PBS.

11. Replace the PBS with $1 \mathrm{~mL} /$ well of phenol red-free medium supplemented with $10 \%$ FBS without any antibiotics.

12. Add the entire $1 \mathrm{~mL}$ of transfection mixture dropwise to each well and incubate the cells overnight at 37 ${ }^{\circ} \mathrm{C}$, in $5 \% \mathrm{CO}_{2}$. 
13. For labeling of the SNAP construct, dilute the appropriate SNAP substrate stock solution in $1 \mathrm{~mL}$ of the medium supplemented with $10 \%$ FBS to obtain a final concentration of $1 \mu \mathrm{M}$.

14. Wash the transfected cells once with PBS and add $1 \mathrm{~mL}$ per well of $1 \mu \mathrm{M}$ SNAP substrate solution. Incubate the cells for 20 min at $37{ }^{\circ} \mathrm{C}$ in $5 \% \mathrm{CO}_{2}$.

15. Wash the cells thrice with phenol red-free medium and add $2 \mathrm{~mL}$ per well phenol red-free medium. Incubate the cells for 30 min at $37^{\circ} \mathrm{C}$ in $5 \% \mathrm{CO}_{2}$.

16. Transfer the coverslips of all samples subsequently into the imaging chamber and wash with $500 \mu \mathrm{L}$ imaging buffer. Add $500 \mu \mathrm{L}$ imaging buffer before moving to the FRET-FCS setup.

\section{Calibration Measurements}

NOTE: The FRET-FCS setup is equipped with a confocal microscope water objective, two laser lines, a Time-Correlated Single Photon Counting (TCSPC) system, two hybrid photomultiplier tubes (PMT) and two avalanche photodiodes (APD) for photon collection and the data collection software. It is very important to align the setup every time before live cell measurements. The detailed setup description can be found in Supplementary Note 2. Both lasers and all detectors (two PMTs and two APDs) are always ON during the measurements, as all measurements need to be conducted under identical conditions. For the calibration measurements, use a coverslip from the same lot on which the cells were seeded, this decreases the variation in collar ring correction.

1. For adjusting the focus, pinhole and collar ring position, place $2 \mathrm{nM}$ green calibration solution on a glass coverslip and switch on the $485 \mathrm{~nm}$ and
$560 \mathrm{~nm}$ laser. Operate laser in Pulsed Interleaved Excitation (PIE) mode ${ }^{16}$.

2. Focus on the solution and adjust the pinhole and collar ring position such that the highest count rate and smallest confocal volume are obtained to get the maximum molecular brightness.

3. Repeat this process for the red channels with $10 \mathrm{nM}$ red calibration solution and a mixture of both, the green and red calibration solution.

4. Place the $10 \mathrm{nM}$ DNA solution on a glass coverslip and adjust the focus, pinhole, and collar ring position such that the cross-correlation between the green and red detection channels is highest, i.e., shows the highest amplitude.

NOTE: Steps 1.2.1 and 1.2.4 might have to be repeated back and forth to find the optimal alignment. Take 3-5 measurements from each calibration solution for $30 \mathrm{~s}-120 \mathrm{~s}$ after the focus, pinhole, and collar ring position have been aligned optimally for the green and red detection channels and the confocal overlap volume.

5. Measure a drop of $d \mathrm{dH}_{2} \mathrm{O}$, the imaging medium, and a non-transfected cell 3-5 times each for $30 \mathrm{~s}-120$ $\mathrm{s}$ to determine the background count rates.

6. Collect the instrument response function with 3-5 measurements for $30 \mathrm{~s}-120 \mathrm{~s}$. This is optional but highly recommended.

3. Live cell measurements

1. Find a suitable cell by illuminating with the mercury lamp and observing through the ocular.

NOTE: Suitable cells are alive showing the typical morphology of the respective adherent cell line. The 
fluorescence of the protein of interest, here a surface receptor, is visible all over the surface. Less bright cells are more suitable than brighter ones due to the better contrast in FCS when a low number of molecules are in focus.

2. Switch on both lasers in PIE mode and focus on the membrane by looking at the maximum counts per second.

NOTE: The laser power might need to be reduced for the cell samples (less than $5 \mu \mathrm{W}$ at objective). This depends upon the used fluorophores and the setup.

3. Observe the auto- and cross-correlation curves of the eGFP or labelled SNAP-tag attached to the $\beta_{2} A R$ in the online preview of the data collection software and collect several short measurements $(\sim 2-10)$ with an acquisition time between $60-180 \mathrm{~s}$. NOTE: Do not excite the cells for a long time continuously as the fluorophores may bleach. However, it will depend on the brightness of each cell, how long the measurements can be, and how many measurements in total can be performed.

\section{Data analysis}

1. Data Export

1. Export the correlation curves, $\mathrm{G}\left(t_{C}\right)$, and count rates, $\mathrm{CR}$, from all measurements.

2. Take care to correctly define the "prompt" and "delay" time windows and use the "microtime gating" option in the data correlation software.

NOTE: In total, three different correlations are required: (1) autocorrelation of the green channel in the prompt time window $\left(A C F_{g p}\right)$, (2) autocorrelation of the red channels in the delay time window
$\left(A C F_{r d}\right)$, and finally (3) the cross-correlation of the green channel signal in the prompt time window with the red channel signal in the delay time window (CCFPIE). The data export is shown step-by-step for different software in Supplementary Note 3.

2. Calibration measurements

1. Use the autocorrelation functions of the green $\left(A C F_{g p}\right)$ and red $\left(A C F_{r d}\right)$ fluorophore solutions, and fit them to a $3 \mathrm{D}$ diffusion model with an additional triplet term if required (eq. 1) to calibrate the shape and size of the confocal detection volume for the two used color channels:

$G_{A C F}\left(t_{c}\right)=b+\frac{1}{N} * \cdot\left(\left(\frac{1}{1+t_{c} / t_{D}}\right)\left(\frac{1}{\sqrt{1+s^{2}\left(t_{c} / t_{D}\right)}}\right)\right)\left[1-a_{R}+a_{R} \cdot \exp \left(-\frac{t_{c}}{t_{R}}\right)\right]$

eq. 1

Here, $b$ is the baseline of the curve, $N$ the number of molecules in focus, $t_{D}$ the diffusion time (in $\mathrm{ms}$ ), and $s=z_{0} / w_{0}$ the shape factor of the confocal volume element. The triplet blinking or other photophysics is described by its amplitude $a_{R}$ and relaxation time $t_{R}$. NOTE: All variables and symbols used within the protocol are listed in Table 1.

2. Use the known diffusion coefficients $D$ for the green $^{26}$ and red calibration standard ${ }^{27}$ and the obtained shape factors sgreen and sred to determine the dimensions (width wo and height $z_{0}$ )

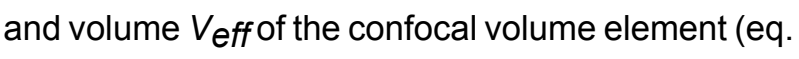
2a-c).

$$
\begin{aligned}
& w_{0}^{2}=4 t_{D} D \quad \text { eq. } 2 \mathrm{a} \\
& z_{0}=s w_{0} \quad \text { eq. } 2 \mathrm{~b} \\
& V_{\text {eff }}=\pi^{3 / 2} z_{0} w_{0}^{2} \quad \text { eq. } 2 \mathrm{c}
\end{aligned}
$$


NOTE: Templates for calculation of the calibration parameter are provided as supplementary files (S7).

3. Calculate the spectral crosstalk $\alpha$ of the green fluorescence signal (collected in channels 0 and 2) into the red detection channels (channel number 1 and 3 ) as a ratio of the background-corrected (BG) signals (eq. 3 ).

$\alpha=\frac{\left(\left(C R_{1, \text { green }}-C R_{1, B G}\right)+\left(C R_{3, \text { green }}-C R_{3, B G}\right)\right)}{\left(\left(C R_{0, \text { green }}-C R_{0, B G}\right)+\left(C R_{2, \text { green }}-C R_{2, B G}\right)\right)} * 100 \%$

eq. 3

4. Determine the direct excitation of the acceptor fluorophore $\delta$ by the donor excitation wavelength by the ratio of the background-corrected count rate of the red calibration measurements in the "prompt" time window (excitation by green laser) to the background-corrected count rate in the "delay" time window (excitation by red laser) (eq. 4).

$\delta=\frac{\left(\left(C R_{1, \text { prompt,red }}-C R_{1, \text { prompt }, B G}\right)+\left(C R_{3, \text { prompt,red }}-C R_{3, \text { prompt }, B G}\right)\right)}{\left(\left(C R_{1, \text { delay,red }}-C R_{1, \text { delay }, B G}\right)+\left(C R_{3, \text { delay,red }}-C R_{3, \text { delay }, B G}\right)\right)} * 100 \%$

eq. 4

5. Calculate the molecular brightness $B$ of both the green and red fluorophores (eq. 5a-b) based on the background-corrected count rates and the obtained number of molecules in focus, $N$, from the $3 D$ diffusion fit (eq. 1):

$$
\begin{aligned}
& B_{\text {green }}=\frac{C R_{\text {corr }, \text { green }, 0}+C R_{\text {corr }, \text { green }, 2}}{N_{\text {green }}} \text { eq. } 5 \mathrm{a} \\
& B_{\text {red }}=\frac{C R_{\text {corr }, \text { red }, 1}+C R_{\text {corr }, \text { red }, 3}}{N_{\text {red }}} \\
& \text { eq. } 5 \mathrm{~b}
\end{aligned}
$$

6. Fit both $A C F_{g p}$ and $A C F_{r d}$ as well as CCFPIE of the double-labeled DNA to the 3D diffusion model (eq. 1). Keep the obtained shape factors, sgreen and sred, constant for $A C F_{g p}$ and $A C F_{r d}$, respectively.
The shape factor for the CCFPIE, SPIE, is usually in between these two values.

NOTE: In an ideal setup, both $V_{\text {eff,green }}$ and $V_{\text {eff, }}$ red would have the same size and overlap perfectly.

7. Determine the amplitude at zero correlation time, $G_{0}\left(t_{C}\right)$, based on the found values of the apparent number of molecules in focus $\left(N_{\text {green }}, N_{\text {red }}\right.$ and NPIE).

8. Calculate the amplitude ratio $r_{G R}$ and $r_{R G}$ for a sample with $100 \%$ co-diffusion of green and red fluorophores (eq. 6). Be aware that NPIE does not reflect the number of double-labeled molecules in focus but reflects only the $1 / G_{0}\left(t_{C}\right)$.

$r_{G R}=\frac{G_{0, C C F}\left(t_{c}\right)}{G_{0, A C F p p}\left(t_{c}\right)}$ and $r_{R G}=\frac{G_{0, C C F}\left(t_{c}\right)}{G_{0, A C F r d}\left(t_{c}\right)} \quad$ eq. 6

3. Live cell experiments

1. For single-labeled constructs, fit the cell samples to an appropriate model. For the shown membrane receptor, diffusion occurs in a bimodal fashion with a short and a long diffusion time. Additionally, the photophysics and blinking of the fluorophores have to be considered:

$G_{A C F, 2 D}\left(t_{c}\right)=b+\frac{1}{N}\left[\frac{a_{1}}{1+t_{c} / t_{d 1}}+\frac{1-a_{1}}{1+t_{c} / t_{d 2}}\right]\left[1-a_{R}+a_{R} \cdot \exp \left(-\frac{t_{c}}{t_{R}}\right)\right]$ eq. 7

Here, $t_{d 1}$ and $t_{d 2}$ are the two required diffusion times, and $a_{1}$ is the fraction of the first diffusion time. NOTE: In contrast to the calibration measurements, in which the free dyes and DNA strands freely diffuse in all directions, the membrane receptor shows only $2 \mathrm{D}$ diffusion along the cell membranes. This difference between $3 \mathrm{D}$ and 2D diffusion is reflected by the modified diffusion term (compare eq. 1), 
where $t_{D}$ in the $2 D$ case does not depend upon the shape factor $s$ of the confocal volume element.

2. Calculate the concentration $c$ of green or red labeled proteins from the respective $N$ and $V_{\text {eff }}$ using basic math (eq. 8):

$c=\frac{N}{V_{e f f} N_{A}} \quad$ eq. 8

where $N_{A}=$ Avogadro's number

3. For N-terminal SNAP label and intracellular eGFP, fit the two autocorrelations $\left(A C F_{g p}\right.$ and $\left.A C F_{r d}\right)$ of the double-labeled sample using the same model as for the single-labeled constructs for the ACFs (eq. 7) and the CCFPIE using a bimodal diffusion model (eq. 9):

$G_{C C F, 2 D}\left(t_{c}\right)=b+\frac{1}{N}\left[\frac{a_{1}}{1+{ }^{t_{c} / t_{d 1}}}+\frac{1-a_{1}}{1+{ }^{t_{c} / t_{d 2}}}\right] \quad$ eq. 9

NOTE: For a global description of the system, all three curves have to be fit jointly: The diffusion term is identical for all three curves and the only difference is the relaxation term for the CCFPIE. As photophysics of two fluorophores is usually unrelated, no correlation term is required. This absence of relaxation terms results in a flat CCFPIE at short correlation times. However, crosstalk and direct excitation of the acceptor due to the donor fluorophore might show false-positive amplitudes and should be carefully checked for using the calibration measurements.

4. Calculate the concentration $c$ of green or red labeled proteins from the respective $N$ and $V_{\text {eff }}$ using equation 8.

5. Estimate the fraction or concentration, $c_{G R}$ or $C_{R G \text {, }}$ of interacting green and red labeled proteins from the cell samples using the correction factors obtained from the DNA samples, the amplitude ratios $r_{G R}$ and $r R G$ of the cell sample and their respective obtained concentrations (eq. 10).

$c_{G R}=r_{G R} \cdot c_{\text {green }}$ and $c_{R G}=r_{R G} \cdot c_{\text {red }} \quad$ eq. 10

6. For C-terminal SNAP label and intracellular eGFP, fit the two autocorrelations $\left(A C F_{g p}\right.$ and $\left.A C F_{r d}\right)$ of the FRET sample as the single-labeled samples (equation 7) and the CCFFRET to a bimodal diffusion model containing an anti-correlation term (equation 11)

$G_{C C F, 2 D}\left(t_{c}\right)=b+\frac{1}{N}\left[\frac{a_{1}}{1+t^{t_{c} / t_{d 1}}}+\frac{1-a_{1}}{1+{ }^{t_{c}} / t_{d 2}}\right]\left[1-a_{f}\right]\left[1-\sum_{i} a_{R i} \cdot \exp \left(-\frac{t_{c}}{t_{R i}}\right)\right]$

eq. 11

where af reflects the amplitude of the total anticorrelation and $a_{R}$ and $t_{R}$ the respective amplitude and relaxation time.

NOTE: In case of anti-correlated fluorescence changes due to FRET, one or several anticorrelation terms might be required (eq. 11), resulting in a "dip" of CCFFRET at low correlation times coinciding with a rise in the two autocorrelations $\left(A C F_{g p}\right.$ and $\left.A C F_{r d}\right)$. However, be aware that photophysics such as triplet blinking might mask the anti-correlation term by dampening the FRET-induced anti-correlation. A joint analysis supplemented with filtered FCS methods might help to unmask the anti-correlation term. Additionally, technical artifacts stemming from dead times in the counting electronics in the nanoseconds range should be excluded ${ }^{16}$. A more detailed step-by-step procedure on how to perform the analysis in ChiSurf ${ }^{28}$ and templates 
for the calculation of confocal volume or molecular brightness are provided on the Github repository (https://github.com/HeinzeLab/JOVE-FCS) and as supplementary files (Supplementary Note 4 and

Supplementary Note 6). Additionally, the pythonscripts for batch export of data acquired with the Symphotime software in .ptu format can be found there.

\section{Representative Results}

Exemplary results of the calibration and live-cell measurements are discussed below. Additionally, the effect of FRET on the cross-correlation curves is demonstrated based on simulated data next to the effect of protein-proteininteraction increasing the CCFPIE amplitude.

\section{PIE-based FCS data export}

In PIE experiments, data are collected in the time-tag timeresolved mode $(T T T R)^{29,30}$. Figure 1B shows the photon arrival time histograms of a PIE measurement of a doublelabeled DNA strand on the described setup (Supplementary Note 1). The setup has four detection channels. The fluorescence emission is first split by polarization in "S" and "P" directions (referring to the perpendicular and parallel plane in which the electric field of a light wave is oscillating).
Secondly, each polarization direction is then split in two color channels (green, red) before detection, resulting in four channels (S-green, S-red, P-green, P-red). In the "prompt" time window, the green fluorophore gets excited, and the signal is detected in both the green and red channels due to FRET. In the delay time window, only the red fluorophore (in the red channel) is visible. Based on the detection channels and "prompt" versus "delayed" time windows, at least five different correlation curves ( 3 autocorrelation curves (ACFs) and 2 cross-correlation curves (CCFs)) can be obtained (Figure 1C-D): (1) green signal in the prompt time window $\left(A C F_{g p}\right),(2)$ red signal in the prompt time window (in case of FRET, $A C F_{r p}$ ), and (3) red signal in the delay time window $\left(A C F_{r d}\right)$. These ACFs report on the protein mobility, photophysics (e.g., triplet blinking) and other time-correlated brightness changes in the fluorophores (e.g., due to FRET). (4) The PIE-based cross-correlation CCFPIE of the green signal in the prompt time window with the red signal in the delay time window allows determining the fraction of codiffusion of the green and red fluorophore ${ }^{16}$. (5) The FRETbased cross-correlation CCFFRET of the green with the red signal in the prompt time window is related to FRETinduced, anticorrelated brightness changes in the green and red signals $31,32,33$. 
A

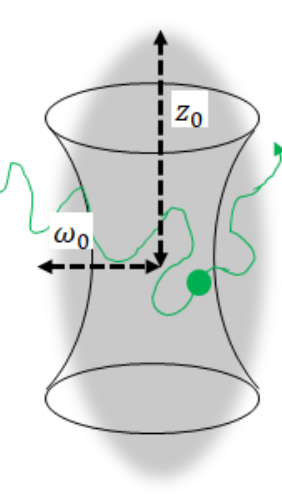

C

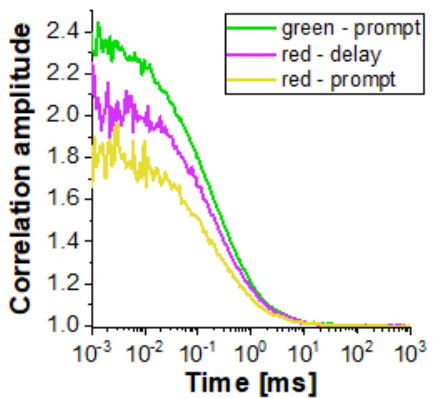

B

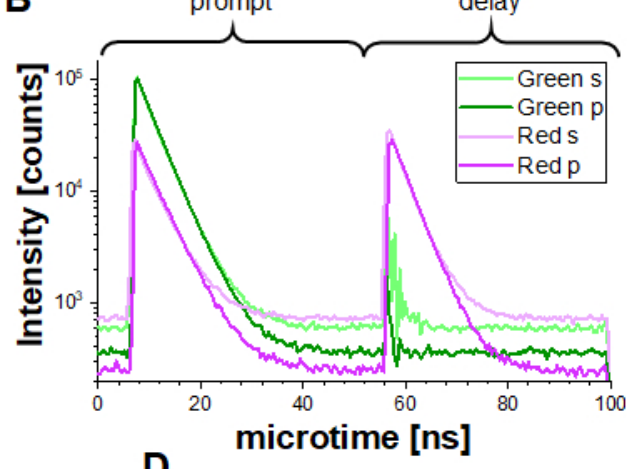

D

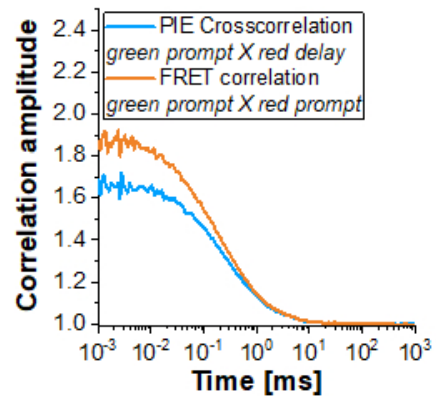

Figure 1: Pulsed-interleaved excitation (PIE) based fluorescence (cross) correlation spectroscopy (F(C)CS). (A) In FCS fluorescently labeled molecules diffuse freely in and out of a (diffraction-limited) focal volume shaped by a focused laser beam that induces fluorescence within this tiny volume. The resulting intensity fluctuations of molecules entering and leaving the volume are correlated and provide information on the mobility of the molecules. (B) In PIE, two different laser lines ("prompt" and "delay") are used to excite the sample labeled with two different fluorophores ("green" and "red"). The time difference between both excitation pulses is adapted to the fluorescence lifetimes of the respective fluorophores so that one has decayed before the other is excited. In the double-labeled sample shown, both fluorophores are sufficiently close to undergo Förster Resonance Energy Transfer (FRET) from the "green" donor fluorophore to the "red" acceptor fluorophore. Thus, red fluorescence emission can be detected in the "prompt" time-window upon excitation of the green donor. In the used setup (Supplementary Note 2), two detectors are used for each color, one oriented parallel to the excitation beam orientation (denoted "p") and the second perpendicular (denoted "s"). (C) Three different autocorrelation functions can be determined in a PIE experiment: Correlation of the i) green channel signals in the prompt time window $\left(A C F_{g p}\right)$, ii) red channel signals in the prompt time window $\left(A C F_{r p}\right)$ and iii) red channel signals in the delay time window $\left(A C F_{r d}\right)$. (D) Two different cross-correlation functions can be determined: iv) The "PIE" cross-correlation (CCFPIE) with green channel signals in the prompt time window correlated with the red channel signals in the delay window, where the amplitude of this curve is related to the co-diffusion of fluorophores; and v) the "FRET" cross-correlation (CCFFRET) with the green channel signals in the prompt time window correlated with the red channel signals in the same prompt window; here the shape of this curve at 
times faster than diffusion is related to the FRET-induced intensity changes. Please click here to view a larger version of this figure.

Calibration

Figure 2A-B shows a calibration measurement of the singly diffusing green and red fluorophores, respectively. Based on a fit with eq. 1 and the known diffusion coefficient $D_{\text {green }}{ }^{26}$ and $D_{\text {red }}{ }^{27}$ the shape $\left(z_{0}\right.$ and $\left.w_{0}\right)$ and size ( $V_{\text {eff }}$ ) of the detection volume are calculated using eq. 2a-c. The fit results from the $A C F_{g p}$ from the green fluorophore and $A C F_{r d}$ from the red fluorophore are summarized in Figure 2C. Both fluorophores show an additional relaxation time constant of $8.6 \mu \mathrm{s}(18 \%)$ and $36 \mu \mathrm{s}(15 \%)$, respectively. The molecular brightness (eq. 5a-b) of the green and red fluorophore amounts to $12.5 \mathrm{kHz}$ per molecule and $2.7 \mathrm{kHz}$ per molecule, respectively.

For a reliable estimation of the confocal volume size and shape as well as the molecular brightness, it is recommended to perform 3-5 measurements per calibration experiments and a joint (or global) fit of all repeats.

The crosstalk $\alpha$ (Figure 2D, eq. 3) and the direct excitation of acceptor by the green laser $\delta$ (Figure 2E, eq. 4) for this fluorophore pair lie at $\sim 15 \%$ and $\sim 38 \%$, respectively.

Copyright $\odot 2021$ JoVE Creative Commons Attribution-NonCommercial-NoDerivs 3.0 Unsorted

jove.com

December $2021 \cdot 178 \cdot$ e $62954 \cdot$ Page 10 of 28

License 

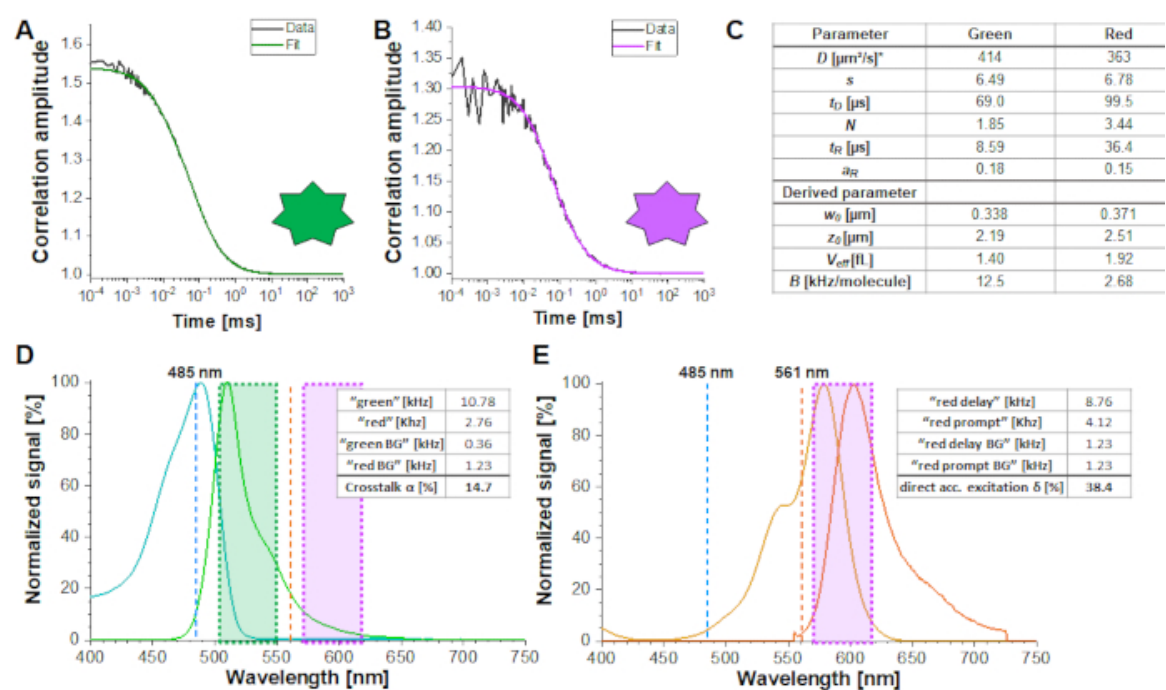

E

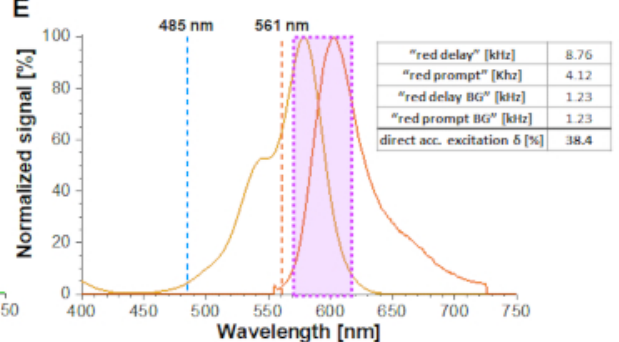

Figure 2: Calibration measurements of freely diffusing green and red calibration standard. (A-B) Representative 60 s measurement of a $2 \mathrm{nM}$ green $(\mathbf{A})$ and a $10 \mathrm{nM}$ red $(\mathbf{B})$ calibration standard measurement fitted to the $3 \mathrm{D}$ diffusion model including an additional relaxation time (eq. 1). The table in panel (C) shows the fit results and the derived parameter based on eq. 2a-c and eq. 5a-b. *Diffusion coefficients were taken from literature ${ }^{26,27}$. (D) Determination of the crosstalk $\alpha$ of the green signal into the red channels (eq. 3). The excitation spectrum of the green standard is shown in cyan, the emission spectrum in green. The excitation laser lines at $485 \mathrm{~nm}$ (blue) and $561 \mathrm{~nm}$ (orange) are shown as dashed lines. Transparent green and magenta boxes show the collected emission range (Supplementary Note 2). (E) Determination of the direct excitation $\delta$ of the red fluorophore by the $485 \mathrm{~nm}$ laser (eq. 4). Color code is identical to (D), light and dark orange show the excitation and emission spectrum of the red standard, respectively. Please click here to view a larger version of this figure.

To determine and calibrate the overlap of the green and red excitation volume, a double-labeled DNA double strand is used (Figure 3A) as described above. Here, the fluorophores are spaced $40 \mathrm{bp}$ apart such that no FRET can occur between the green and red fluorophores attached to the ends of the DNA double strands. Figure $3 \mathrm{~B}$ shows the autocorrelations from both fluorophores in green $\left(A C F_{g p}\right)$ and magenta $\left(A C F_{r d}\right)$ and the PIE-cross correlation, CCFPIE, in cyan. Please note that for CCFPIE, the signal in the green channels in the prompt time window is correlated with the signal in the red channels in the delay time window ${ }^{16}$.
Here, an average diffusion coefficient for the DNA strand of $D_{D N A}=77 \mu \mathrm{m}^{2} / \mathrm{s}$ is obtained. More details on the calculation can be found in the step-by-step protocol, Supplementary Note 4. This value is obtained by inserting the calibrated green and red detection volumes size (Figure 2) and the respective diffusion times of $A C F_{g p}$ and $A C F_{r d}$ of the DNA strand (Figure $\mathbf{3 C}$ ) into equation $2 \mathrm{a}$. Next, using the obtained correction values $r_{G R}$ and $r_{R G}$ and using eq. 6 later on, the amount of co-diffusion, i.e., double-labeled molecules (or protein complexes in case of co-transfection of two different proteins) can be determined from the cell samples. 

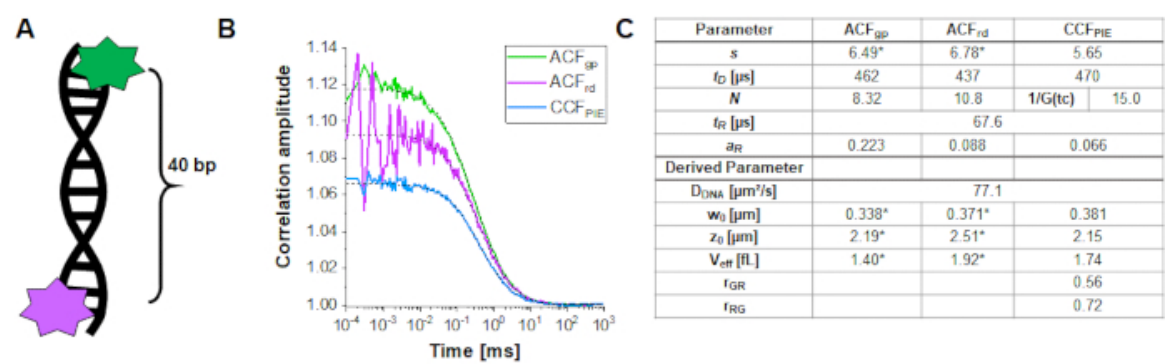

Figure 3: Calibration of the green-red overlap volume using a DNA sample. (A) The DNA strand used for calibration carries a green and a red calibration fluorophore, with a distance of $40 \mathrm{bp}$ in between. The interdye distance must be sufficiently large to exclude FRET between the fluorophores. (B) Representative 60 s measurement of a $10 \mathrm{nM}$ DNA solution. Autocorrelations from both fluorophores in green $\left(A C F_{g p}\right.$, green standard) and magenta $\left(A C F_{r d}\right.$, red standard) and the PIE-crosscorrelation, CCFPIE, in blue. The table in panel (C) shows the fit results based on the 3D Diffusion model including an additional relaxation term (eq. 1) and the derived parameter diffusion coefficient of DNA, DDNA (eq. 2a), the size and shape of the overlap volume (eq. 2a-c) and the correction ratios $r_{G R}$ and $r_{R G}$ (eq. 6). Please note the values for the green and red detection volume (labeled with *) were taken from the fit of the individual fluorophores shown in Figure 2. Please click here to view a larger version of this figure.

\section{Live-cell experiments}

In the following section, the analysis of live-cell experiments for different $\beta_{2} A R$ constructs is presented. As $\beta_{2} A R$ is a membrane protein, its diffusion is largely limited to a twodimensional diffusion (Figure 4A) along the cell membrane (except for transport or recycling processes to or from the membrane ${ }^{2}$. With the restriction to the $2 \mathrm{D}$ diffusion the shape factor $s=z_{0} / w_{0}$ in eq. 1 becomes obsolete resulting in a simplified diffusion model (eq. 9).

Single-labeled constructs: $\quad \beta_{2} A R-I L 3-e G F P$ and NTSNAP- $\beta_{2} A R$

Figure 4 shows exemplary measurements of the singlelabel construct $\beta_{2} A R$-IL3-eGFP (Figure 4B), where eGFP is inserted into the intracellular loop 3, and the construct NTSNAP-B2AR (Figure 4C), where the SNAP tag is conjugated to the $\mathrm{N}$-terminus of $\beta_{2} \mathrm{AR}$. The SNAP tag is labeled with a membrane-impermeable SNAP surface substrate. The representative curves show the average of 4-6 repeated measurements with acquisition times of $120-200$ s each. The respective autocorrelations $A C F_{g p}$ and $A C F_{r d}$ of the eGFP and SNAP signal are fitted to a bimodal, two-dimensional diffusion model (eq. 9). In terms of fast dynamics, eGFP shows only the expected triplet blinking at $t_{R 1} \sim 9 \mu$ s wile the SNAP signal requires two relaxation times, one at the typical triplet blinking time of $t_{R 1} \sim 5 \mu$ and a second one at $t_{R 2} \sim$ $180 \mu s$.

The molecular brightness of the fluorophores in living cells is $0.8 \mathrm{KHz}$ (eGFP) and $1.7 \mathrm{kHz}$ (SNAP) per molecule under the given excitation conditions (eqs. 5a-b). The concentration of the labeled $\beta_{2} A R$ constructs incorporated in the cell membrane should be in the nano-molar range and can be determined by the average number of molecules (eq. 9, 
Figure 4C) and the size of the respective confocal volume for the green and red channel (Figure 2) using eq. 8.

A

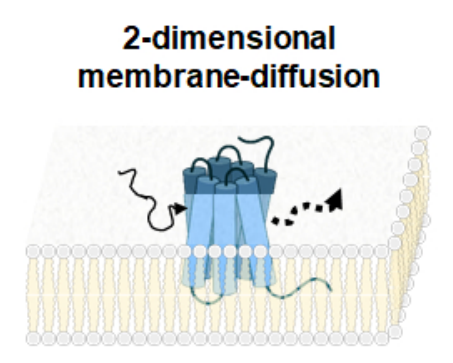

C

\begin{tabular}{|c|c|c|}
\hline Parameter & ACF $_{\mathrm{gp}}(\mathrm{eGFP})$ & ACF $_{\mathrm{rd}}$ (SNAP) \\
\hline $\boldsymbol{t}_{\mathrm{D} 1}[\mathrm{~ms}]$ & 118 & 48.9 \\
\hline $\mathbf{a}_{1}$ & 0.542 & 0.489 \\
\hline $\boldsymbol{t}_{\mathrm{D} 2}[\mathrm{~ms}]$ & 1.88 & 2.72 \\
\hline $\boldsymbol{N}$ & 5.86 & 35.3 \\
\hline $\boldsymbol{t}_{\boldsymbol{R} 1}[\boldsymbol{\mu} \mathrm{s}]$ & 8.7 & 4.7 \\
\hline $\mathbf{a}_{\boldsymbol{R} 1}$ & 0.252 & 0.120 \\
\hline $\boldsymbol{t}_{\boldsymbol{R} 2}[\boldsymbol{\mu} \mathrm{s}]$ & & 181 \\
\hline $\mathbf{a}_{\boldsymbol{R} 2}$ & & 0.110 \\
\hline Derived Parameter & & \\
\hline B [kHz/molecule] & 0.80 & 1.72 \\
\hline $\mathbf{c}[\mathrm{nM}]$ & 4.30 & 67.5 \\
\hline
\end{tabular}

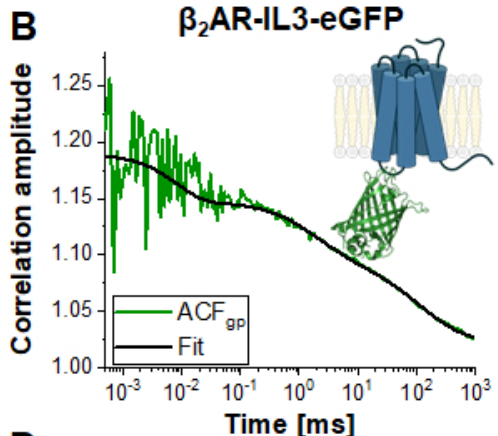

D

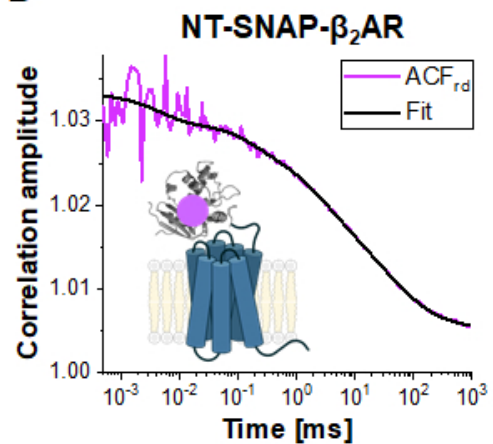

Figure 4: Representative measurement of single-label constructs. (A) In this study, the membrane receptor $\beta_{2} A R$ was used as an example. In contrast to the fluorophores and DNA strand used for calibration, which could freely float through the detection volume, membrane proteins diffuse mainly laterally along the membrane, described as 2-dimensional diffusion. (B, D) $A C F_{g p}$ and $A C F_{r d}$ of the single-label constructs $\beta_{2}$ AR-IL3-eGFP (B) and NT-SNAP- $\beta_{2} A R(D)$. Shown is the average of 4-6 measurements each collected for 120 - $200 \mathrm{~s}$. The table in panel (C) shows the fit results of the data to the bimodal twodimensional diffusion model including additional relaxation terms (eq. 7). Please click here to view a larger version of this figure.

\section{Double-labeled construct: NT-SNAP- $\beta_{2} A R-I L 3-e G F P$}

In the double-labeled construct NT-SNAP-B2AR-IL3-eGFP (short NT-SNAP), eGFP is inserted into the intracellular loop 3 , and, the SNAP tag conjugated to the $\mathrm{N}$-terminus of $\beta_{2} A R$ (Figure 5A). In this configuration, the eGFP is on the inner side of the membrane and the SNAP on the outer side with too large distances for FRET. In an ideal case, this construct would show $100 \%$ co-diffusion of the green and red fluorophore, and no FRET signal. Figure 5B-D shows two measurements of the NT-SNAP in two cells on two different measurement days. Fitting the $A C F_{g p}$ and $A C F_{r d}$ of the "better" measurement shown in Figure 5B with eq. 7 and the CCFPIE with eq. 9, reveals 50- 60 molecules in focus for the $A C F_{g p}$ and $A C F_{r d}$, whereas $N_{a p p}$, thus $1 / G_{0}\left(t_{C}\right)$ $\sim 114$ for the CCFPIE (Figure 5C). The concentration of labeled receptors lies in the $\sim 100 \mathrm{nM}$ range as determined 
with eq. 8. To determine the average concentration of doublelabeled molecules, first, the ratio of $\mathrm{G}_{0}\left(t_{C}\right)$ (represented by $\left.1 / N_{(a p p)}\right)$ of the CCFPIE to $A C F_{g p}$ and $A C F_{r d}$, respectively, is calculated (eq. 6). Next, these values, rGRcell=0.43 and $r R G$ cell $=0.53$ are compared to the values obtained from the DNA measurement $\left(r G R, D N A=0.51\right.$ and $r_{R G, D N A}=0.79$ on this measurement day). Using the rule of proportions, a rGRcell= 0.43 from the $A C F_{g p}$ of the eGFP signal reflects to a fraction of co-diffusion ( $r_{G R c e l / I}$ IR,DNA) of 0.84 , where for the other case of $A C F_{r d}$ of the SNAP substrate signal, this value amounts to 0.67 . The average concentration of the double-labeled NT-SNAP construct can finally be calculated based on eq. 10. In contrast, in the measurement shown in Figure 5D from a different day, the concentration of receptors is quite low and the data very noisy such that the fit range is limited up to $\sim 10 \mu \mathrm{s}$. In addition, only a low amount of codiffusion is observed (15 - 26\%).

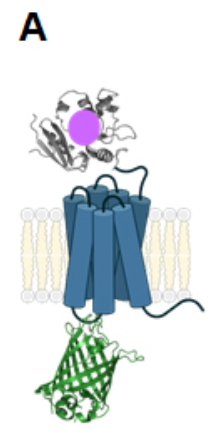

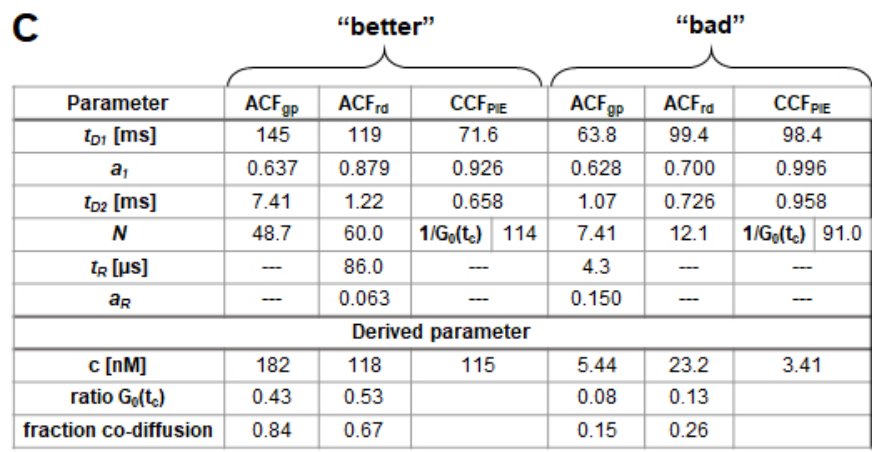

\section{B}
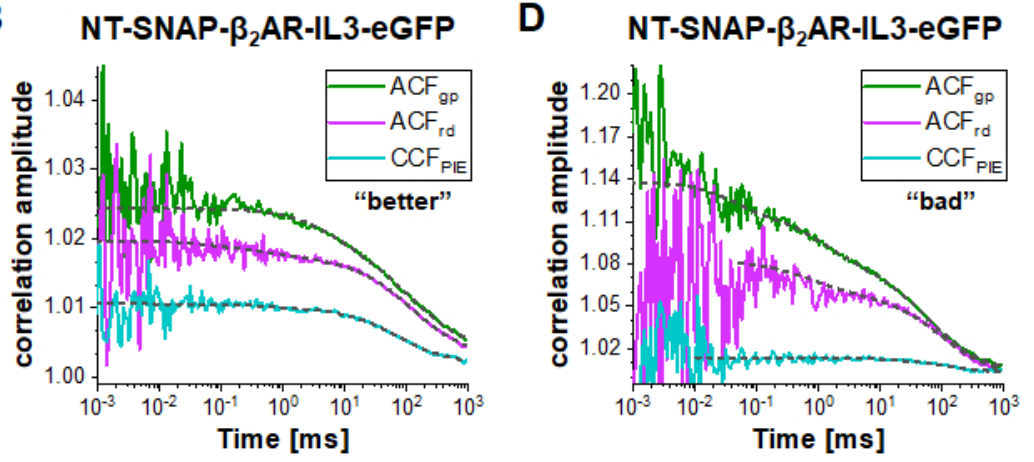

Figure 5: Double-labeled NT-SNAP-ß2AR-IL3-eGFP construct. (A) In the double labeled construct, the eGFP is inserted into the intracellular loop 3 and the SNAP tag attached to the N-terminus of $\beta_{2} A R$ (NT-SNAP). (B, D) $A C F_{g p}, A C F_{r d}$ and CCFPIE of two measurements of the double-labeled construct. The data is fit to a bimodal two-dimensional diffusion model (eq. 9, CCFPIE) and including additional relaxation terms (eq. 7, $A C F_{g p}$ and $\left.A C F_{r d}\right)$. The table in panel (C) shows the fit results and the derived parameter concentration (eq. 8), the ratio of the correlation amplitude at zero correlation time $\left(G_{0}\left(t_{C}\right)\right)$ and the fraction of co-diffusing molecules (eq. 10). Please note that the measurements were acquired on different days, thus slightly different factor for the amplitude correction were used (B: rGR,DNA $=0.51$ and $r R G, D N A=0.79 ; \mathrm{D}: r_{G R, D N A}=0.51$ and $\left.r_{R G, D N A}=0.56\right)$. Please click here to view a larger version of this figure. 
Double-labeled construct undergoing FRET: $\beta_{2} A R-I L 3-$ eGFP-CT-SNAP

In the double-labeled construct $\beta_{2}$ AR-IL3-eGFP-CT-SNAP (Figure 6A), the eGFP is inserted into the intracellular loop 3 identical to the NT-SNAP- $\beta_{2}$ AR-IL3-eGFP construct with the SNAP tag attached to the C-terminus. Here, both labels are on the same side of the cells' plasma membrane, so that the fluorophores are in close vicinity so that FRET occurs as indicated by the quenched eGFP lifetime (Supplementary Note 5). Considering the flexibility of relatively unstructured protein regions like the $\mathrm{C}$-terminus ${ }^{34}$ and at least two different protein conformations of $\mathrm{GPCRs}^{35}$, "high FRET" (HF) or "low FRET" (LF), dynamic changes in the FRET efficiency due to eGFP-SNAP distance changes could be observed and identified by an anticorrelation term in the CCFFRET (orange curve in Figure 6B). FRET fluctuations have been shown to be anticorrelated as the receptor can only be in one state at a time, either HF or LF. Joint (or global) fit of all five correlation curves (Figure 6B) reveals $\sim 70 \%$ of slowly diffusing molecules at $\sim 100 \mathrm{~ms}$ while the rest diffuses with $\sim 1 \mathrm{~ms}$. All autocorrelations and CCFFRET show relaxation terms at $37 \mu \mathrm{s}$ and $3 \mu \mathrm{s}$; those correlations dominated by red signal $\left(A C F_{r p}, A C F_{r d}\right.$ and $\left.C C F_{F R E T}\right)$ show an additional slow component $\sim 50 \mathrm{~ms}$ (Figure 6C).

FRET-induced changes on the CCFFRET under different conditions (Figure 6D) are demonstrated by a series of simulations of a two-state system with a fluctuation time of $70 \mu$ s between LF and HF states. Upon switching from the LF to the HF state, changes in the anticorrelated signal are observed in the prompt time window: The green signal decreases and the red signal increases (vice versa for the HF -> LF switching). If HF-LF switching occurs on timescales faster than the diffusion time, in other words during the residence time of the molecule in the focus, the rate can be derived from the anticorrelation in the $\operatorname{CCF}_{F R E T^{6,31,36}}$. Please note that dynamic processes slower than the diffusion time cannot be observed in FCS.

In this demonstration, two different FRET scenarios were assumed, showing either a moderate or maximal change in FRET efficiency between the two states. The simulations were performed using Burbulator ${ }^{37}$ and consider absence or presence of triplet blinking and increasing amount of donor crosstalk into the red channels. The diffusion term was modeled as a bimodal distribution with $30 \%$ of fast diffusing molecules at $t_{D 1}=1 \mathrm{~ms}$ and the rest of the molecules diffusing slowly with $t_{D 2}=100 \mathrm{~ms}$. In total, $10^{7}$ photons were simulated in a 3D Gaussian-shaped volume with $w_{0}=0.5 \mu \mathrm{m}$ and $z_{0}=1.5 \mu \mathrm{m}$, a box size of 20 , and NFCS $=0.01$.

Figure 6E-F shows the simulation results for the FRETinduced cross-correlation CCFFRET for moderate (Figure 6E) and maximal FRET contrast (Figure 6F) in the absence (solid lines) and presence of triplet blinking (dashed lines). The FRET-induced anti-correlation can easily be seen in Figure 6F. The "dampening" effect upon adding an additional triplet state reduces the correlation amplitude (Figure 6EF) 38,39 

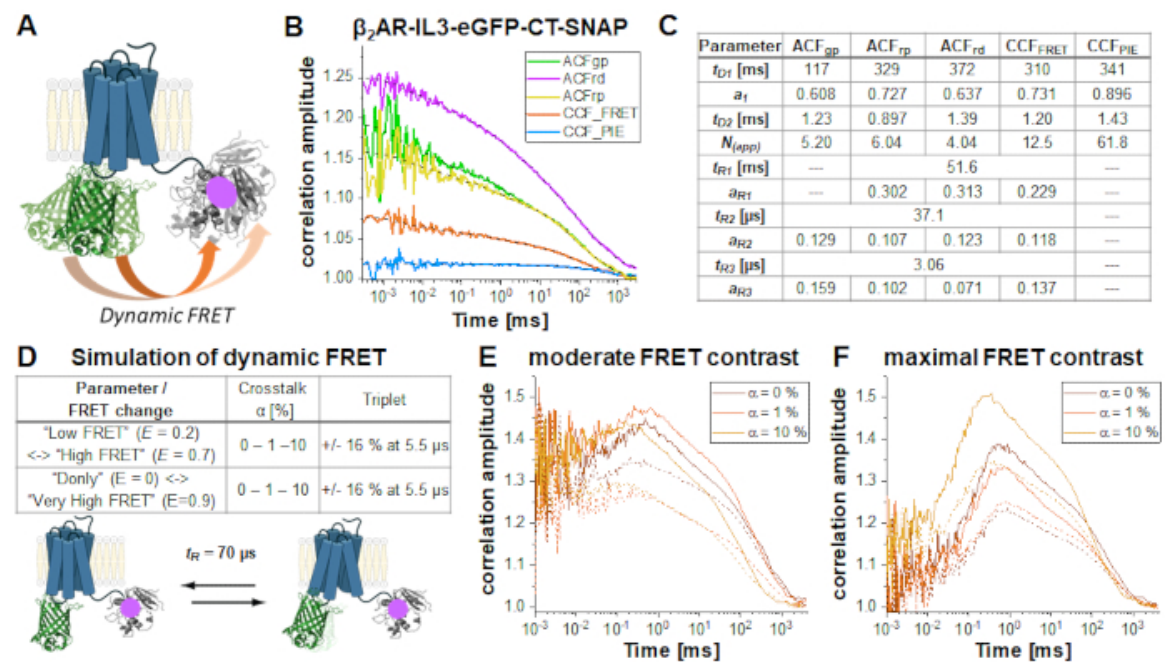

Figure 6: Simulation of double-labeled sample showing dynamic FRET. (A) Double-labeled $\beta_{2} A R$ with an eGFP inserted into the intracellular loop 3 and a C-terminal SNAP tag. Both fluorophores are close enough to undergo FRET and show changes in the FRET efficiency if the receptor undergoes protein dynamics. (B) Autocorrelation $\left(A C F_{g p}, A C F_{r p}\right.$ and $A C F_{r d}$, fit with eq. 7) and cross-correlation curves (CCFFRET (eq. 7) and CCFPIE (eq. 9)) of an example measurement. Table in panel (C) shows the fit results. (D-F) To show the influence of experimental parameter on the expected, FRETinduced anticorrelation term, 12 simulations were performed, in which the change in the FRET efficiency (small or large), different amount of donor crosstalk into the acceptor channels $(0 \%, 1 \%$ or $10 \%)$ and the absence and presence of triplet blinking were modeled. The equilibrium fraction of both FRET-states was assumed to 50:50 and their exchange rates adjusted such that the obtained relaxation time $t_{R}=70 \mu \mathrm{s}$. More details on the simulations see in the text. (E) CCFFRET of the simulation results with a moderate FRET contrast and in the absence of crosstalk (dark orange), 1\% crosstalk (orange) and $10 \%$ crosstalk (light orange). Solid lines show results in the absence of triplet, dashed lines in the presence of triplet. (F) CCFFRET of the simulation results with maximal FRET contrast. The color code is identical to (E). Please click here to view a larger version of this figure.

However, in the simulation most similar to the experimental conditions $(\alpha=10 \%, 15 \%$ triplet blinking and moderate FRET contrast, dashed yellow line in Figure 6E), the anticorrelation term is nearly diminished. Figure 7 shows the result of analyzing this simulated data using the information encoded in the photon arrival time histograms (i.e., the fluorescence lifetime) by means of Fluorescence Lifetime
Correlation Spectroscopy (FLCS) $)^{17,19}$ or species-filtered FCS (fFCS) ${ }^{18}$. Here, the fluorescence lifetimes of the known HF and LF species (Figure 7A) are used to generate weights or "filters" (Figure 7B) which are applied during the correlation procedure. In the obtained species-auto- and cross-correlation curves (Figure 7C-D) the anticorrelation can be clearly observed. 

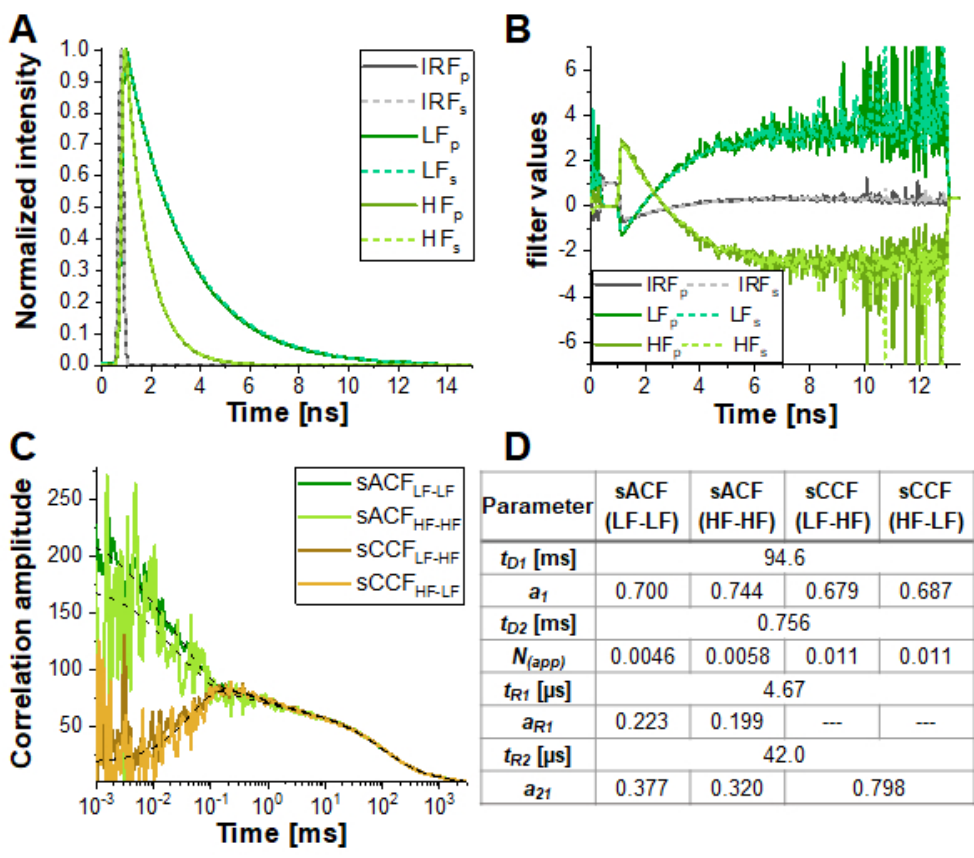

\begin{tabular}{|c|c|c|c|c|}
\hline Parameter & $\begin{array}{c}\text { sACF } \\
\text { (LF-LF) }\end{array}$ & $\begin{array}{c}\text { sACF } \\
\text { (HF-HF) }\end{array}$ & $\begin{array}{c}\text { sCCF } \\
\text { (LF-HF) }\end{array}$ & $\begin{array}{c}\text { sCCF } \\
\text { (HF-LF) }\end{array}$ \\
\hline$t_{D 1}[\mathrm{~ms}]$ & \multicolumn{4}{|c|}{94.6} \\
\hline$a_{1}$ & 0.700 & 0.744 & 0.679 & 0.687 \\
\hline$t_{D 2}[\mathrm{~ms}]$ & \multicolumn{4}{|c|}{0.756} \\
\hline$N_{(a p p)}$ & 0.0046 & 0.0058 & 0.011 & 0.011 \\
\hline$t_{R 1}[\mu \mathrm{s}]$ & \multicolumn{4}{|c|}{4.67} \\
\hline$a_{R 1}$ & 0.223 & 0.199 & - & - \\
\hline$t_{R 2}[\mu \mathrm{s}]$ & \multicolumn{4}{|c|}{42.0} \\
\hline$a_{21}$ & 0.377 & 0.320 & & 798 \\
\hline
\end{tabular}

Figure 7: Lifetime-filtered FCS can help to uncover the protein dynamics based fluctuations in FRET efficiency in samples with high crosstalk, significant triplet blinking or other photophysical or experimental properties masking the FRET-induced anticorrelation in the CCFFRET. Here, the approach is shown exemplary for the data shown in Figure 6E for the simulation containing $10 \%$ crosstalk and $5 \%$ triplet blinking. (A) Normalized fluorescence intensity decay patterns for the two FRET-species (light and dark green for high and low FRET, respectively) and the IRF (grey). The pattern for the parallel detection channel is shown in solid lines, dashed lines for the perpendicular detection channel. (B) The weighting function or "filter" were generated based on the patterns shown in $(\mathbf{A})$, color code is identical to $(\mathbf{A})$. Please note that only the signal in the green detection channels, and thus the FRET-induced donor quenching, is considered here. (C) Four different species-selective correlations are obtained: species-autocorrelations of the low FRET state (sACF $L F-L F$, dark green) and the high FRET state (sACFHF-HF, light green), and the two species-crosscorrelations between the low FRET to the high FRET state ( $s C C F L F-H F$, dark orange) and vice versa (sCCFHF-LF, orange). The sCCF clearly shows the anticorrelation in the $\mu$ s-range. Dashed black lines show the fits. SACF were fit with eq. 9 and SCCF with eq. 11. Table in panel (D) shows the fit results. Please click here to view a larger version of this figure.

\section{CCFPIE amplitude to study Protein-Protein Interaction} (PPI)

Finally, a common use case for PIE-based FCS in live cells is to study the interaction between two different proteins. Here, the read-out parameter is the amplitude of the CCFPIE, or more precisely the ratio of the autocorrelation amplitudes $A C F_{g p}$ and $A C F_{r d}$ to the amplitude of CCFPIE. To show the effect of increasing co-diffusion on CCFPIE, simulations have been performed based on the two single-labeled constructs, $\beta_{2} A R-I L 3-e G F P$ and NT-SNAP- $\beta_{2} A R$ (Figure 8A). Figure 
8B shows how the amplitude of CCFPIE increases when the fraction of co-diffusing molecules changes from $0 \%$ to $100 \%$. Please note that a $1 \%$ crosstalk of green signal into the red channels in the delay time window was added with the diffusion components otherwise modeled as shown above.
A

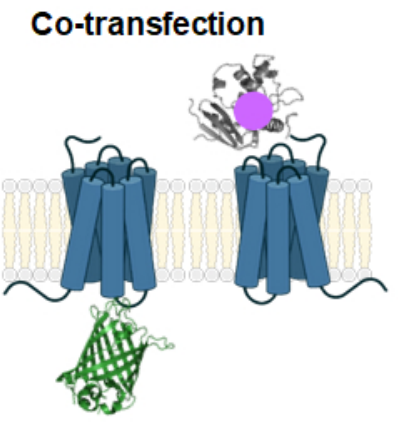

B

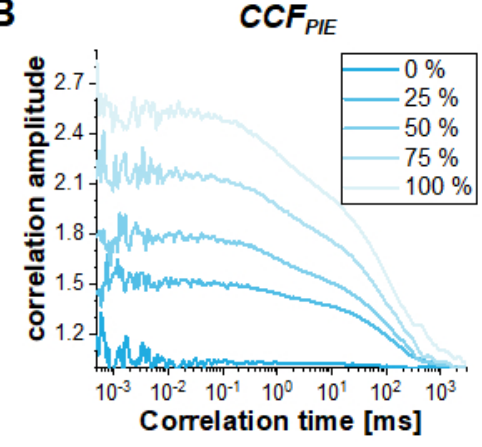

Figure 8: The CCFPIE can be used to study the interaction of two proteins. (A) Here, a co-transfection study of $\beta_{2} A R-$ IL3-eGFP with NT-SNAP- $\beta_{2}$ AR (carrying a "red" SNAP-label) was simulated. (B) For an increasing amount of co-diffusing molecules $\left(0 \%\right.$ (dark blue) -> 100\% (light blue)) the amplitude $\mathrm{G}\left(t_{C}\right)$ increases. The diffusion term was again modeled as a bimodal distribution with $30 \%$ of fast diffusing molecules at $t_{D 1}=1 \mathrm{~ms}$ and the rest of the molecules diffusing slowly with $t_{D 2}$ $=100 \mathrm{~ms}$. Additionally, $1 \%$ crosstalk of green signal into the red delay time window was added. Please click here to view a larger version of this figure. 


\begin{tabular}{|c|c|}
\hline Symbol & Meaning (common unit) \\
\hline$\alpha$ & crosstalk of the green fluorophore after green excitation into the red detection channels (\%) \\
\hline$a_{1}$ & fraction of first diffusion component in bimodal diffusion model of membrane receptors \\
\hline af & total amplitude of the anticorrelation term \\
\hline aR & amplitude of photophysics /triplet blinking \\
\hline $\mathrm{b}$ & baseline / offset of a correlation curve \\
\hline B & molecular brightness of a fluorophore ((kilo-)counts per molecule and second) \\
\hline BG & background (e.g. from an appropriate reference sample: ddH2O, buffer, untransfected cell etc.) \\
\hline c & concentration \\
\hline CR & count rate (KHz or (kilo-) counts per second) \\
\hline$\delta$ & direct excitation of the red fluorophore after green excitation (\%) \\
\hline $\mathrm{D}$ & diffusion coefficient $\left(\mu \mathrm{m}^{2} / \mathrm{s}\right)$ \\
\hline $\mathrm{G}\left(\mathrm{t}_{\mathrm{C}}\right)$ & correlation function \\
\hline $\mathrm{N}$ & number of molecules in focus \\
\hline $\mathrm{N}_{\mathrm{A}}$ & Avogadro's number $\left(6.022 * 10^{23} \mathrm{Mol}^{-1}\right)$ \\
\hline rGR, rRG & amplitude ratio of green or red autocorrelation function to the PIE-based cross-correlation function \\
\hline s & shape factor of confocal volume element \\
\hline$t_{C}$ & correlation time (usually in millisecond) \\
\hline tD & diffusion time (usually in millisecond or microsecond) \\
\hline tR & relaxation time of photophysics (usually in microsecond) \\
\hline $\mathrm{t} T$ & relaxation time of triplet blinking (usually in microsecond) \\
\hline wo & half-width of confocal volume element $(\mu \mathrm{m})$ \\
\hline$z_{0}$ & half-height of confocal volume element $(\mu \mathrm{m})$ \\
\hline
\end{tabular}

Table 1: List of variables and abbreviations. For the use of symbols and definition in fluorescence and FRET experiments, the guidelines of the FRET community ${ }^{40}$ are recommended. 


\section{SUPPLEMENTARY FILES:}

\section{SuppNote1_Coverslip cleaning.docx Please click here to} download this File.

\section{SuppNote2_Confocal Setup.docx Please click here to download this File.}

\section{SuppNote3_Data export.docx Please click here to download this File.}

\section{SuppNote4_FCCS calibration analysis using ChiSurf.docx Please click here to download this File.}

\section{SuppNote5_Fluorescence lifetime histograms.docx} Please click here to download this File.

\section{S6_Scripts.zip Please click here to download this File.}

\section{S7_Excel_templates.zip Please click here to download this} File.

\section{Discussion}

FCS techniques in GPCRs allow the mobility and interactions of receptors inside live cells to be assessed ${ }^{41}$. The advantage of the FRET-FCS technique is that, along with mobility, the conformational dynamics of GPCRs can be investigated. However, performing FRET-FCS in live cells is challenging and requires cells which show low (or maximally moderate) expression of the fluorescently labeled protein of interest, a well-calibrated setup and a good pipeline to analyze data. Here, first the critical points in sample preparation and experimental procedure are discussed concerning the biological, spectroscopic, and technical points of view.

Critical experimental steps include minimizing the background and autofluorescence (by using extensively cleaned coverslips and phenol-red free media), the optimization of transfection conditions (e.g., amount of plasmid DNA and time after transfection) to achieve low expression levels and efficient labeling. Of course, it is also vital to ensure that the function of the labeled protein is not hampered. Thus, in live-cell experiments, the decision for the labeling strategy and label position is often made in favor of fluorescent proteins or SNAP/CLIP tag attached to the flexible $\mathrm{N}$ - or $\mathrm{C}$-terminus ${ }^{42,43}$. Alternative labeling strategies like inserting an unnatural amino acid with a reactive side chain for labeling with an organic fluorophore have been emerging in the last years ${ }^{44}$.

For dual-color PIE-FCS, where only the interaction of two molecules of interest is to be investigated, the fluorophores can be selected from a large variety of established fluorescent proteins or SNAP/CLIP substrates. Here, spectroscopy-wise the goal should be to select a pair such that little crosstalk or direct acceptor excitation occur. Additionally, the selected fluorophores should be photostable and show little or no bleaching under the chosen experimental conditions. It is recommended to select fluorophores in the red spectral range as (1) the autofluorescence background from the cell is reduced and (2) the excitation light is of longer wavelength, thus less phototoxic ${ }^{14}$. Photobleaching can be minimized by conducting a so-called "power series" first, in which the laser power is increased stepwise, and the molecular brightness is observed. The optimal excitation intensity range lies in the linear range of the results ${ }^{45}$. 
If the two labels are supposed to report also on protein conformational dynamics through FRET then the choice of available fluorophores is more restricted. Here, the possible minimal/maximal distance between the two fluorophores should be estimated beforehand e.g., based on available structures or molecular size, and a fluorophore pair selected with a reasonable Förster radius $R_{0}$ such that FRET can actually occur ${ }^{20}$.

Here, eGFP and a SNAP tag were chosen for labeling, and the SNAP tag was labeled with either an intracellular or a membrane-impermeable surface substrate. The spectra are similar to the ones shown in Figure 2C-D. This combination of fluorophores shows high crosstalk of the eGFP into the red detection channels and direct acceptor excitation of the SNAP substrate by the green excitation in the prompt time window and results in a significant "false" signal in the red channels in the prompt time window. Ideally, both values, cross talk and direct acceptor excitation, should not exceed $5 \% 5,6,38$. However, with a Förster radius of $57 \AA$, it is ideally suited to probe the distance between the labels in the $\beta_{2} A R$-IL3-eGFPCT-SNAP construct as can be evaluated from the quenched eGFP lifetime (Supplementary Note 5).

Technically, as for any fluorescence spectroscopy experiment, the device should be well aligned and should possess suitable excitation sources, emission filter, and sensitive detectors. To avoid artifacts from detector afterpulsing on the $\mu$ s timescale, at least two detectors of each color should be present, which can be crosscorrelated. In modern time-correlated single photon counting electronics, the dead time of the detection card in the ns time range hardly plays a role due to the independent routing channels, however, it might be checked as proposed by Müller et al ${ }^{16}$ provided the time range of interest lies in the sub- $\mu \mathrm{s} / \mathrm{ns}$ time range. Additionally, for even higher time resolutions in the ps range, each detection channel should be doubled, i.e., four detectors per color should be used, to also bypass detector dead times ${ }^{2,15,29,46}$. While the average fluorescence lifetime can be estimated using nonpolarized fluorescence detection, for analysis of the distance ( distribution) between the fluorophores the emission has to be collected polarization-dependent. This is due to the fact that the efficiency of the energy transfer in FRET relies on the orientation of the two fluorophores. More detailed information can be found here $20,28,47$. Finally, in PIE experiments, the distance between the prompt and delay pulse is critical and should be chosen such that the fluorescence intensity of the fluorophores has been largely decayed (Figure 1B). A common rule is to place the two pulses $5 x$ the fluorescence lifetime apart, i.e. for eGFP with a fluorescence lifetime of 2.5 ns the distance should be $12.5 \mathrm{~ns}$ at minimum ${ }^{22}$.

After having detailed all considerations for the experimental procedure, the data and its analysis is discussed in more detail. As mentioned in the protocol section, the alignment of the setup must be checked daily, including the analysis of the calibration measurements. The data shown in Figure 2A-C e.g., shows an additional relaxation component in the 8-40 $\mu$ s range. Typical triplet blinking of the green calibration fluorophore is known to occur in the 2-10 $\mu$ s range $13,15,48$. The slow relaxation component required in all curves of the DNA sample (Figure $\mathbf{3 C}$ ), too slow for actual triplet blinking, might stem from interactions of the DNA with the fluorophores ${ }^{39}$. However, this component would not be expected in CCFPIE, and most likely stems from residual crosstalk. Thus, it is highly advisable to perform the analysis of the calibration samples directly prior to proceeding to the cell experiments to judge the quality of the day's alignment. 
The proper calibration of the confocal overlap volume requires a sample with $100 \%$ co-diffusion of the green and red label. Here, fluorescently labeled double stranded DNA is used. Both DNA strands can be tailored to have the desired fluorophores at the required distance from each other. The designed strands can be annealed with high yield. However, Good Laboratory Practice advises checking the integrity and labeling degree of the DNA strands by agarose gel electrophoresis and measuring the absorption spectrum. Also, the yield of the double-stranded assembly should be checked as this calibration measurement critically relies on the assumption that there is a $100 \%$ co-diffusion of the green with the red label. In case the assumption is not valid, correction factors might have to be applied ${ }^{16,22}$. In the calibration measurements shown in Figure 2 and Figure 3, a detection volume of $1.4 \mathrm{fL}$ and $1.9 \mathrm{fL}$ in the green and red channel were obtained, respectively. This size difference is expected for a setup with nearly diffraction-limited excitation volumes (Supplementary Note 2). Under this condition, the size of the excitation volume scales with the excitation wavelength. This in turn explains the different correlation amplitudes observed in Figure 3B. The derived correction factors $r_{G R}=0.56$ and $r_{R G}=0.72$ correct for this size discrepancy and potential non-perfect overlap of the two excitation volumes ${ }^{3,4}$.

Figure 4, Figure 5, Figure 6, and Figure 7 showcase the workflow of a PIE-F(C)CS based study aimed toward understanding conformational protein dynamics. First, the two single-labeled constructs $\beta_{2}$ AR-IL3-eGFP and NT-SNAP$\beta_{2} A R$ serve as controls to characterize the fluorophore properties in cells in the absence of the respective other fluorophore (Figure 4). Next, the double-labeled construct NT-SNAP-B2AR-IL3-eGFP carrying a SNAP-tag facing the cell exterior and an eGFP on the cytoplasmic side serves as a "100\% co-diffusion" control (Figure 5). The last construct, $\beta_{2}$ AR-IL3-eGFP-CT-SNAP, carries both fluorophores on the cytoplasmic side and close enough together to undergo FRET. Here, again a $100 \%$ co-diffusion would be expected in tandem with anti-correlated intensity fluctuations in the green and red channels signal in the prompt time window, i.e., after donor excitation, due to protein dynamics influencing the FRET efficiency ${ }^{31,32,33}$. This dynamics might show up as anti-correlation in the CCFFRET (Figure 6-7).

All GPCR $\beta_{2} A R$ constructs show bimodal diffusion on the cell membrane (Figure 4A). Whereas the $\beta_{2}$ AR-IL3-eGFP shows only the expected triplet blinking (Figure 5B) ${ }^{13,15}$, NTSNAP- $\beta_{2} A R$ shows an additional slow relaxation time (Figure 5C-D). It is likely that $t_{R 2}$ might stem from unbound SNAP substrate. This could be elucidated by further experiments, e.g., by also measuring the diffusion and photophysical properties of the used SNAP substrate in an aqueous solution. Of note, a straightforward experiment to differentiate between diffusion and relaxation times is to change the pinhole of the confocal setup, i.e., increasing the effective volume: While the diffusion times increase with increasing effective volumes, relaxation terms are unaltered ${ }^{13}$. When determining the concentration of fluorescent protein (FP) based on the fit results, be aware that FPs in general undergo a maturation process, in which finally the chromophore is formed ${ }^{12}$. This maturation time may differ from FP to FP in addition to photophysics that depends on the local chemical environment ${ }^{13,15}$. Thus, the actual protein concentration present in the sample reported by FCS is usually underestimated, which can be corrected if the fraction of non-fluorescent FPs can be determined in the experiment. Finally, it is advisable to check the fluorophore spectra in live cells to correct the values for $\alpha$ and $\delta$, if required, as most fluorophores react sensitive to 
their environment ${ }^{13,15,48}$. The background to subtract is determined by the signal collected in non-transfected cells. Additionally, the autocorrelation of the respective other color channel and the CCFPIE should be checked to be able to identify false signals (Supplementary Note 4 - Figure 30).

The two measurements from the NT-SNAP-ß32AR-IL3-eGFP (Figure 5D), where the fluorophores are located on different sides of the membrane, were acquired on different days and shows the importance of statistics in time-resolved single molecule fluorescence. Here, the different results may be due to the different degree of labeling: In one cell the higher degree of labeling and averaging of measurements resulted in relatively low noise (Figure 5B), while from the other cell, only two measurements could be collected (Figure 5A). Beyond collecting a sufficient amount of data, it is critical to evaluate the results timely, and maybe optimize the labeling strategy. When designing the experiments, it is important to remember that FRET is sensitive, but limited to distances up to $10 \mathrm{~nm}$ and "blind" otherwise. In our case, this "blindness" is indicated by the unaltered eGFP fluorescence lifetime (Supplementary Note 5). In the $\beta_{2}$ AR-IL3-eGFP-CT-SNAP construct (Figure 6A), FRET can be pinpointed from the quenched eGFP lifetime (Supplementary Note 5). However, no anticorrelation term is observed (Figure 6B), which means that FRET is either not fluctuating or at a time scale slower than the diffusion time. Up to three additional relaxation terms are required in $A C F_{g p}, A C F_{r p}, A C F_{r d}$ and $C C F_{F R E T}$ (Figure 6C). The slow component in $A C F_{r p}, A C F_{r d}$ and $C C F_{F R E T}$ might be due to acceptor bleaching and, of course, influences the obtained value of the slow diffusion found in these curves ( $350 \mathrm{~ms}$ compared to $117 \mathrm{~ms}$ in $\left.A C F_{g p}\right)$. $t_{D}$ in the red channel is supposed to be slightly larger than in the green channel due to the differently sized confocal volumes (Figure 2) - but only by a factor comparable to the size difference. The very fast relaxation time of $3 \mu$ seflects the triplet blinking of the fluorophores ${ }^{13,15,48}$, whereas the slower relaxation time of 37 us might be due to FRET: Similarly, as FRET induces an anticorrelation in the CCFFRET, positive correlations are expected in the autocorrelations ${ }^{31,32,33}$. The presence of this term as "positive" in CCFFRET and its presence in the $A C F_{r d}$ might be explained with the high crosstalk and should be further elucidated. Note that the CCFPIE is flat at short correlation times as expected.

On the other hand, it should be noted that the occurrence of FRET in a system of interest leads to non-linear effects on the correlation curves ${ }^{6}$. The molecular brightness e.g., of a molecule scales into the correlation amplitude squared and each FRET-state (and the always present molecules without an active receptor) shows different molecular brightness. Indeed, FRET decreases the apparent concentration of green molecules detected (i.e., increases $A C F_{g p}$ amplitude) and the number of red molecules (determined from redprompt) is overestimated ${ }^{5}$. Both effects influence the amount of interaction derived from both CCFFRET and CCFPIE. However, global analysis as shown e.g. for the intramolecular dynamics of Calmodulin 31,32 or Syntaxin ${ }^{33}$ can reveal the protein dynamics. When carefully calibrated, the average FRET efficiency may be extracted from the relative CCFPIE and ACF amplitudes ${ }^{22}$, whereas the limiting states might be determined from the analysis of the donor fluorescence lifetime distribution ${ }^{33}$.

Considering the fact that in live cell experiments with large fluorophores like eGFP the FRET contrast is likely to be even lower than assumed for the simulations shown in Figure 6 and that the direct excitation of the acceptor was not added in the simulation, might explain why the identification of the anticorrelation in live cell experiments is very challenging. 
A promising analysis alternative relies on harvesting the information encoded in the photon arrival time histograms (Figure 1B) accessible due to the time-correlated single photon counting data collection 29,30 . If the fluorescence lifetime ( patterns) of the two (or more) (FRET) species inside the sample are known (Figure 7A), "filter" or weights can be chosen which are applied during the correlation process (Figure 7B) $)^{17,18,19}$. The correlation curves thus obtained, no longer represent the correlation of detection channels but rather the auto- or cross-correlations between two different (FRET) species, thus renamed to species-ACF (SACF) or species-CCF (sCCF). Applying this approach to the simulated data with moderate FRET contrast, high crosstalk and triplet blinking recovers the anticorrelation term (Figure 7C-D). However, it should be noted that relaxation times can be obtained but the relationship to amplitude is $\operatorname{lost}^{18}$. This approach has been applied previously in live cell experiments e.g. to study the interaction of EGFR with its antagonist ${ }^{49}$ or to separate the fluorescence from proteins attached to eGFP variants with exceptionally short and long fluorescence lifetimes ${ }^{50}$.

While PIE-based FRET measurements in purified proteins are largely used to study protein dynamics ${ }^{36} 22$, in live cells it focuses on understanding protein-protein interactions. This approach has been applied to study the regulation of MAP kinase activity in yeast ${ }^{51}$ or to resolve the interaction of membrane proteins with their cytosolic binding partner as summarized in this recent article ${ }^{52}$. Here, complications may arise when significant crosstalk of green fluorophores is still present in the delay time window of the red channels or red signal in the green channels in the prompt time window. The former might be caused by an insufficient delay of the red pulse with respect to the green pulse while both effects stem from too strongly overlapping excitation and emission spectra of the chosen fluorophores. It is recommended to check the respective single labeled constructs carefully and correct for false-positive CCFPIE amplitudes, especially in cells where autofluorescence with very short fluorescence lifetime might be another complicating factor 22 .

To conclude, the FRET-FCS approach described here has great potential to understand protein-protein interactions and protein dynamics in live cells at near physiological concentrations. In this protocol, the focus was laid on the required calibration measurements and the necessary quantitative analysis to be performed during live cell measurements. To this end, different live cell measurements were shown complemented with simulations. The simulations provide the general understanding here as parameter could be varied systematically with tailored fit models that describe the specific mobility and photophysical properties of the respective data. The analysis was performed with open-source software tools with an extensive step-by-step protocol and easy-to-adapt templates. Finally, the technical advancements, and thus the availability of ready-to-buy stable PIE-FCS systems together with the spread of opensource software for data analysis will make this technique more and more accessible for a larger research community to eventually unravel protein interaction and dynamics in live cells with highest sensitivity.

\section{Disclosures}

The authors have no conflicts to declare.

\section{Acknowledgments}

This project was supported by the Deutsche Forschungsgemeinschaft (SFB/TR 240, project number 374031971, Project INF) to J. B. and K.G.H. 
We thank the Rudolf Virchow Center for financial support and Core Unit Fluorescence Imaging for technical support. Additionally, we thank Ashwin Balakrishnan for thorough proof-reading.

\section{References}

1. Hess, S. T., Huang, S. H., Heikal, A. A., Webb, W. W. Biological and chemical applications of fluorescence correlation spectroscopy: A review. Biochemistry. 41 (3), 697-705 (2002).

2. Haustein, E., Schwille, P. Ultrasensitive investigations of biological systems by fluorescence correlation spectroscopy. Methods. 29 (2), 153-166 (2003).

3. Bacia, K., Kim, S. A., Schwille, P. Fluorescence crosscorrelation spectroscopy in living cells. Nature Methods. 3 (2), 83-89 (2006).

4. Bacia, K., Schwille, P. Practical guidelines for dual-color fluorescence cross-correlation spectroscopy. Nature Protocols. 2 (11), 2842-2856 (2007).

5. Kohl, T., Heinze, K. G., Kuhlemann, R., Koltermann, A., Schwille, P. A protease assay for two-photon crosscorrelation and FRET analysis based solely on fluorescent proteins. Proceedings of the National Academy of Sciences. 99 (19), 12161-12166 (2002).

6. Sahoo, H., Schwille, P. FRET and FCS--friends or foes? Chemphyschem. 12 (3), 532-541 (2011).

7. Wang, Y., Wang, G., Moitessier, N., Mittermaier, A. K. Enzyme Kinetics by Isothermal Titration Calorimetry: Allostery, Inhibition, and Dynamics. Frontiers in Molecular Bioscience. 7583826 (2020).
8. Yanase, Y. et al. Surface plasmon resonance for cell-based clinical diagnosis. Sensors (Basel). 14 (3), 4948-4959 (2014).

9. Freedberg, D. I., Selenko, P. Live cell NMR. Annual Review of Biophysics. 43 171-192 (2014).

10. Nishida, N., Ito, Y., Shimada, I. In situ structural biology using in-cell NMR. Biochimica et Biophysica Acta General Subjects. 1864 (2), 129364 (2020).

11. Schwille, P., Meyer-Almes, F. J., Rigler, R. Dualcolor fluorescence cross-correlation spectroscopy for multicomponent diffusional analysis in solution. Biophysical Journal. 72 (4), 1878-1886 (1997).

12. Balleza, E., Kim, J. M., Cluzel, P. Systematic characterization of maturation time of fluorescent proteins in living cells. Nature Methods. 15 (1), 47-51 (2018).

13. Haupts, U., Maiti, S., Schwille, P., Webb, W. W. Dynamics of fluorescence fluctuations in green fluorescent protein observed by fluorescence correlation spectroscopy. Proceedings of the National Academy of Sciences. 95 (23), 13573-13578 (1998).

14. Icha, J., Weber, M., Waters, J. C., Norden, C. Phototoxicity in live fluorescence microscopy, and how to avoid it. BioEssays. 39 (8), 1700003 (2017).

15. Widengren, J., Mets, U., Rigler, R. Photodynamic properties of green fluorescent proteins investigated by fluorescence correlation spectroscopy. Chemical Physics. 250 (2), 171-186 (1999).

16. Müller, B. K., Zaychikov, E., Bräuchle, C., Lamb, D. C. Pulsed interleaved excitation. Biophysical Journal. 89 (5), 3508-3522 (2005). 
17. Böhmer, M., Wahl, M., Rahn, H.-J., Erdmann, R., Enderlein, J. Time-resolved fluorescence correlation spectroscopy. Chemical Physics Letters. 353 (5), 439-445 (2002).

18. Felekyan, S., Kalinin, S., Sanabria, H., Valeri, A., Seidel, C. A. M. Filtered FCS: Species Auto- and CrossCorrelation Functions Highlight Binding and Dynamics in Biomolecules. Chemphyschem. 13 (4), 1036-1053 (2012).

19. Kapusta, P., Wahl, M., Benda, A., Hof, M., Enderlein, J. Fluorescence lifetime correlation spectroscopy. Journal of Fluorescence. 17 (1), 43-48 (2007).

20. Algar, W. R., Hildebrandt, N., Vogel, S. S., Medintz, I. L. FRET as a biomolecular research tool-understanding its potential while avoiding pitfalls. Nature Methods. 16 (9), 815-829 (2019).

21. Elson, E. L., Magde, D. Fluorescence Correlation Spectroscopy .1. Conceptual Basis and Theory. Biopolymers. 13 (1), 1-27 (1974).

22. Hendrix, J., Lamb, D. C. Pulsed interleaved excitation: principles and applications. Methods Enzymol. 518 205-243 (2013).

23. Magde, D., Elson, E. L., Webb, W. W. Fluorescence correlation spectroscopy. II. An experimental realization. Biopolymers. 13 (1), 29-61 (1974).

24. Thompson, N. L. in Topics in Fluorescence Spectroscopy. (ed J. R. Lakowicz) 337-378 Plenum Press, (1991).

25. Cole, N. B. Site-specific protein labeling with SNAPtags. Current protocols in protein science. 73 30.31.31-30.31.16 (2013).
26. Petrásek, Z., Schwille, P. Precise measurement of diffusion coefficients using scanning fluorescence correlation spectroscopy. Biophysical Journal. 94 (4), 1437-1448 (2008).

27. Siegel, A. P., Baird, M. A., Davidson, M. W., Day, R. N. Strengths and Weaknesses of Recently Engineered Red Fluorescent Proteins Evaluated in Live Cells Using Fluorescence Correlation Spectroscopy. International Journal of Molecular Sciences. 14 (10), 20340-20358 (2013).

28. Peulen, T. O., Opanasyuk, O., Seidel, C. A. M. Combining Graphical and Analytical Methods with Molecular Simulations To Analyze Time-Resolved FRET Measurements of Labeled Macromolecules Accurately. The Journal of Physical Chemistry B. 121 (35), 8211-8241 (2017).

29. Wahl, M., Rahn, H. J., Gregor, I., Erdmann, R., Enderlein, J. Dead-time optimized time-correlated photon counting instrument with synchronized, independent timing channels. Review of Scientific Instruments. 78 (3), 033106 (2007).

30. Wahl, M. et al. Scalable time-correlated photon counting system with multiple independent input channels. Review of Scientific Instruments. 79 (12), 123113 (2008).

31. Price, E. S., Aleksiejew, M., Johnson, C. K. FRETFCS Detection of Intralobe Dynamics in Calmodulin. The Journal of Physical Chemistry B. 115 (29), 9320-9326 (2011).

32. Price, E. S., DeVore, M. S., Johnson, C. K. Detecting intramolecular dynamics and multiple Forster resonance energy transfer states by fluorescence correlation spectroscopy. The Journal of Physical Chemistry B. 114 (17), 5895-5902 (2010). 
33. Margittai, M. et al. Single-molecule fluorescence resonance energy transfer reveals a dynamic equilibrium between closed and open conformations of syntaxin 1. Proceedings of the National Academy of Sciences. 100 (26), 15516-15521 (2003).

34. Cherezov, V. et al. High-resolution crystal structure of an engineered human beta2-adrenergic $\mathrm{G}$ protein-coupled receptor. Science. 318 (5854), 1258-1265 (2007).

35. Manglik, A. et al. Structural Insights into the Dynamic Process of beta2-Adrenergic Receptor Signaling. Cell. 161 (5), 1101-1111 (2015).

36. Olofsson, L. et al. Fine tuning of sub-millisecond conformational dynamics controls metabotropic glutamate receptors agonist efficacy. Nature Communications. 5 (1), 5206 (2014).

37. Kalinin, S., Valeri, A., Antonik, M., Felekyan, S., Seidel, C. A. Detection of structural dynamics by FRET: a photon distribution and fluorescence lifetime analysis of systems with multiple states. The Journal of Physical Chemistry B. 114 (23), 7983-7995 (2010).

38. Hom, E. F. Y., Verkman, A. S. Analysis of coupled bimolecular reaction kinetics and diffusion by twocolor fluorescence correlation spectroscopy: Enhanced resolution of kinetics by resonance energy transfer. Biophysical Journal. 83 (1), 533-546 (2002).

39. Widengren, J., Schweinberger, E., Berger, S., Seidel, C. A. M. Two new concepts to measure fluorescence resonance energy transfer via fluorescence correlation spectroscopy: Theory and experimental realizations. Journal of Physical Chemistry A. 105 (28), 6851-6866 (2001).
40. Hellenkamp, B. et al. Precision and accuracy of single-molecule FRET measurements-a multi-laboratory benchmark study. Nature Methods. 15 (9), 669-676 (2018).

41. Briddon, S. J., Kilpatrick, L. E., Hill, S. J. Studying GPCR Pharmacology in Membrane Microdomains: Fluorescence Correlation Spectroscopy Comes of Age. Trends in Pharmacological Sciences. 39 (2), 158-174 (2018).

42. Barak, L. S. et al. Internal trafficking and surface mobility of a functionally intact beta2-adrenergic receptor-green fluorescent protein conjugate. Molecular Pharmacology. 51 (2), 177-184 (1997).

43. Calebiro, D. et al. Single-molecule analysis of fluorescently labeled G-protein-coupled receptors reveals complexes with distinct dynamics and organization. Proceedings of the National Academy of Sciences. 110 (2), 743 (2013).

44. Nikić, I., Kang, J. H., Girona, G. E., Aramburu, I. V., Lemke, E. A. Labeling proteins on live mammalian cells using click chemistry. Nature Protocols. 10 (5), 780-791 (2015).

45. Robert, T. Y., Haibing, T. Measuring protein dynamics in live cells: protocols and practical considerations for fluorescence fluctuation microscopy. Journal of Biomedical Optics. 19 (9), 1-24 (2014).

46. Felekyan, S. et al. Full correlation from picoseconds to seconds by time-resolved and time-correlated single photon detection. Review of Scientific Instruments. 76 (8), 083104 (2005). 
47. Forster, T. ${ }^{*} Z$ wischenmolekulare Energiewanderung Und Fluoreszenz. Annalen Der Physik. 2 (1-2), 55-75 (1948).

48. Widengren, J., Mets, U., Rigler, R. Fluorescence Correlation Spectroscopy of Triplet-States in Solution - a Theoretical and Experimental-Study. Journal of Physical Chemistry. 99 (36), 13368-13379 (1995).

49. Chen, J., Irudayaraj, J. Fluorescence lifetime cross correlation spectroscopy resolves EGFR and antagonist interaction in live cells. Analytical Chemistry. 82 (15), 6415-6421 (2010).

50. Stefl, M., Herbst, K., Rubsam, M., Benda, A., Knop, M. Single-Color Fluorescence Lifetime Cross-Correlation Spectroscopy In Vivo. Biophysical Journal. 119 (7), 1359-1370 (2020).

51. Maeder, C. I. et al. Spatial regulation of Fus3 MAP kinase activity through a reaction-diffusion mechanism in yeast pheromone signalling. Nature Cell Biololy. 9 (11), 1319-1326 (2007).

52. Christie, S., Shi, X., Smith, A. W. Resolving Membrane Protein-Protein Interactions in Live Cells with Pulsed Interleaved Excitation Fluorescence Cross-Correlation Spectroscopy. Accounts of Chemical Research. 53 (4), 792-799 (2020). 